\title{
Research on Prevention and Treatment of Chronic Fatigue Syndrome through Aerobic Exercise
}

\author{
Hongbing Zeng ${ }^{1}$, Chengmei Wang ${ }^{1}$
}

\author{
${ }^{1}$ Department of Military Sports, Chongqing Vocational Institute of Engineering, Chongqing, 402260, \\ China
}

Key word: Aerobic exercise, Chronic fatigue syndrome, Immunologic system, Exercise immunity.

\begin{abstract}
Chronic fatigue syndrome was found and understood in recent years. It is different from t he chronic fatigue emerged with a healthy human body. A large number of study found it was possi ble with a wide range of pathogens such as viruses, bacteria and might cause infection-related immu ne disorder. Aerobic exercises through a variety of biological mechanisms promote health. To discu ss the intrinsic relationship between aerobic exercise and the immune of body and the role of mecha nisms which provide a basis for scientifically and effectively carry out prevention and control for ch ronic fatigue syndrome.
\end{abstract}

\section{Introduction}

Chronic fatigue syndrome (CFS) is formally named by Centers for Disease Control and Prevention in 1987, is the neurosis that concerns damage to brain and neurological function caused on the basis of chronic fatigue, is a kind of disease that results in loss of normal living ability of healthy people due to their cryptogenic long-lasting and recurrent symptoms such as generalized weakness, low-grade fever, pharyngalgia, lymphatic pain, headache, muscle pain, arthralgia and psychoneurosis.[1] CFS has a significant impact on patients' work and life and has increasingly attracted high attention of the medical field. However, people in our country have little knowledge of this disease; therefore, it is necessary to remind people of strengthening their self-health care consciousness and being vigilant against sickness from constant overwork. This paper focuses on researching the regulation of body immune system through proper amount of aerobic exercise, and consequently discusses the relation between aerobic exercise and prevention and treatment of chronic fatigue syndrome.

\section{Impact of chronic fatigue syndrome on immune function}

Researches show that most CFS patients suffer from immune system dysfunction, thus CFS is also called "chronic fatigue immune dysfunction syndrome" (CFIDS)[2]. CFS patients may have low activity in natural killer (NK) cells[3], positive antinuclear antibodies (ANA), abnormal immune globulins, increased immune complex in blood, mononuclear lymphocyte dysfunction, lymphocyte subpopulation and its dysfunction[4], and abnormal increase in cytokines such as interleukin 1 ( IL $1 \beta)$, interleukin $6(\mathrm{IL}-6)$, tumor necrosis factor $\alpha(\mathrm{TNF}-\alpha)$ and interferon $\gamma($ IFN $-\gamma)[5]$. The changes in number and ratio of T-cell subsets result in decline in immune function of body cells, so that viruses entering the body cannot be eliminated but lurk in the body for a long time and cause pharyngalgia. Toxins produced by bacteria in patients' bodies induce the generation of anandamide (AEA); recently, it is discovered in the experiment of using delta hemolysin to induce experimental animal's fever and pain that, if the action of AEA is interrupted simultaneously, the aforesaid symptoms will not appear; it is speculated that CFS patients are related to AEA system dysfunction. It is hereby implied that CFS patients' feeling of fatigue is a common clinical manifestation caused by various immune disorders or infectious diseases. 


\section{Impact of aerobic exercise on body immune function}

Aerobic exercise is a kind of exercise, the energy supply of which is achieved mainly through sugar and fat metabolism when oxygen absorbed meets the body's oxygen demand. As the stimulus that causes responsive reaction of immune system, aerobic exercise directly stimulates the body's immune system, makes it feel the environmental changes in the body during exercise through its own recognition system and consequently triggers a series of immune reactions, including producing specific antibodies, enhancing the activity of NK cells and increasing leucocytes and sensitized lymphocytes, so as to maintain the new environmental balance in the body. Regular aerobic exercise can enhance the body's immune function, resist diseases and improve life quality [6].

\section{Impact on T-lymphocytes}

T-lymphocytes are important for adjusting the body's immunoreaction and directly reflect the state of immune function of body cells. Balanced CD4 and CD8 in lymphocyte subpopulations are the basis of stable immune function of the body; when the normal proportion of CD4 and CD8 changes, the body's immune condition will change accordingly[7]. Current researches show that T-lymphocytes are very sensitive to the intensity and time of exercise. According to Landmart's report, exercise at 25\%, 50\% and 75\%VO2max intensities for 3 minutes respectively can increase the number of lymphocyte subpopulations, and long-time moderate-intensity exercise (at 60\%VO2max for 2 hours) can also increase lymphocytosis[8]. In the exercise state, nerve, internal secretion and internal environment will have different impacts on immune system. The immune system itself will also have different changes accordingly. With the body's gradual adaption to load, the body reaction caused by the stress resisting and adapting to the exercise is increasingly small under the same load, the suppression degree declines gradually and the suppression time gets shorter, so as to accelerate recovery of T-lymphocytes. With the body's gradual adaption to aerobic exercise, the body's counter-shock phase during "stress" period and the release of various stress hormones lowering the activity of T-lymphocytes decline, and the immunosuppression reduces, so as to gradually accelerate the recovery of activity of T-lymphocytes.

\section{Impact on NK cells}

NK cells play an important role in the first line of defense system that resists virus infection in the body. It is generally thought that the decline in activity of NK cells is related to decline in immune function. Researches on rats made by Yan Jun et al. show that exercise at medium and low loads can effectively increase the number of NK cells in the body, and that the medium-load exercise has a better effect [9]. Wang Fengmei [10] et al. made researches on immune function of elderly women after their aerobic exercise (Table 1):

Table 1. Changes in Quantity of NK Cells before and after Exercise

\begin{tabular}{|c|c|c|c|c|}
\hline \multicolumn{5}{|c|}{ Control Group } \\
\hline Aerobic Group & Before Experiment & After Experiment & Before Exercise & After Exercise \\
\hline Before exercise & $12.6 \pm 2.1$ & $12.9 \pm 2.2$ & $10.2 \pm 2.4$ & $13.4 \pm 2.7$ \\
\hline After exercise & $21.6 \pm 2.6 *$ & $18.7 \pm 4.2 *$ & $22.0 \pm 4.4 *$ & $23.1 \pm 4.4^{*}$ \\
\hline
\end{tabular}

Note: * $\mathrm{P}<0.05$ for comparison before and after high-intensity exercise

It can be seen from Table 1 that the number of NK cells in the quiet state of aerobic group before high-intensity motion after exercise increases significantly $(\mathrm{P}<0.05)$, but the number of NK cells after high-intensity motion before and after exercise has no significant variation. Aerobic exercise can increase NK cells of the body in the quiet state, but has little impact on quantity of NK cells after high-intensity motion. According to the existing reports, impacts of exercise on NK cells mainly include two aspects: firstly, it causes redistribution of circulating lymphocyte subpopulations to change the percentage of NK cells; secondly, it changes the activity of NK cells to adjust killing capability of NK cells from the cell level. It is generally thought that exercise intensity is the key factor to influence the recruitment level of peripheral blood NK cells. Short-time high-intensity acute 
exercise will often cause the recruitment of a large amount of peripheral blood NK cells, and the number of NK cells can increase by $40 \% \sim 100 \%$ within the first few minutes of the exercise; however, after a certain period of time, the concentration of NK cells will decline and even get lower than that before the exercise, and the function of NK cells will also decline. Thus it can be seen that an appropriate amount of regular exercise can enhance the function of NK cells, and that excessive exercise may cause immunosuppression.

\section{Impact on immune globulins}

IgA, IgG and IgM in serum are 3 kinds of important immune globulins that have the humoral immune function in the body; IgG is the Ig that is highest at content in serum, is produced mainly for secondary immune response of the body and is the "main force" for the body to resist infection; IgM is the antibody first appeared in primary humoral immune response, and is the "spearhead" for anti-infection immunity of the body; IgA has such functions as toxin neutralization. Therefore, detecting the levels of IgG, IgM and IgA in serum will contribute to understanding the functions of complex Ig tissues and organs as well as the anti-infection immunity of the body, so as to evaluate the humoral immunity state of the body [11].

\section{Prevention and treatment of chronic fatigue syndrome through aerobic exercise}

At present, there are no specific methods to treat the chronic fatigue syndrome, but exercise at appropriate intensity can facilitate recovery of CFS patients. Researches made by Samoilovych[12] et al. on physical exercise of students suffering from chronic fatigue syndrome show that physical exercise can effectively improve the chronic fatigue syndrome.

From the perspective of exercise immunology, Tang Shuang et al. have analyzed the impact of chronic fatigue syndrome on activity of NK cells of 40 mice selected for research and divided into 4 groups, each of which has 10 mice. Group I is the normal control group, and groups II, III and IV are the CFS control group, CFS exercise group 1 and CFS exercise group 2 respectively. The exhaustive swimming test is adopted to establish the CFS mouse model. After exercise at different intensities, MTT and ELISA methods are adopted respectively to detect the activity of NK cells in spleens of mice (as shown in Fig. 2).

High-intensity acute exercise will often cause recruitment of a large amount of peripheral blood NK cells; but after a certain period of time, the level of NK cells will decline. Long-period high-intensity acute exercise will cause decline in concentration and function of NK cells of mice, while long-term moderate aerobic exercise can increase the level and activity of NK cells of mice. Proper exercise will contribute to CFS recovery. Thus it is concluded that overloaded exercise will cause immune dysfunction of CFS patients. Some scholars concluded through researches that, according to the discovery by means of muscle biopsy after overloaded exercise of sportsmen suffering from chronic fatigue syndrome, aerobic metabolism damage caused by chronic fatigue syndrome did not increase the oxygen consumption of the patients, while keeping on exercise can increase the oxygen consumption. Therefore, suitable aerobic exercise such as walking can relieve the symptoms of CFS patients and improve the state of body immune dysfunction.

\section{Expectations}

Sub-health has become one of the focuses of the global society. The result of a global investigation made by the World Health Organization shows that healthy people accounts for $20 \%$ only, and that $75 \%$ of people are in the transition state between health and suffering from diseases, which is called the third state by WHO, namely the sub-healthy state. CFS patients are a special presentation of sub-healthy state. Causes and pathogenesis of CFS are unknown, the current treatment of CFS just focuses on alleviating the symptoms and improving the functions. CFS is closely related to many factors, while immune dysfunction is an important link resulting in CFS and playing a critical role in 
its generation and development. Therefore, enhancing the body's immune function is an important means of CFS prevention and treatment.

Although CFS cause-unknown, psychiatric symptoms are principal, and mental and social factors have important influence on CFS. Social and psychological factors influence the body's autonomic nerves system, endocrine system, neurotransmitter and immune system, consequently influence the environmental stability in the body and break the body's defense system, and finally cause diseases. Aerobic exercise is a kind of physical exercise under the condition of guaranteeing sufficient oxygen absorption of the body; as a way of mental intervention, the role of reducing psychological pressure of physical exercise has been confirmed. The impact of aerobic exercise on physical and mental health effect lies in the fact that aerobic exercise can satisfy exercisers' physical and psychological needs and make them become adaptive. On one hand, appropriate aerobic exercise acts on central nervous system, generating positive psychological stress for individuals to make them transfer their negative emotions and obtain positive emotions; on the other hand, individuals' suitable action responses under favorable psychological stress can act on immune system through internal secretion and peripheral nervous system, finally improving the immune function.

\section{References}

[1] Fukuda K, Straus S E, Hickie I, et al. The chronic fatigue syndrome: a comprehensive approach to its definition and study. Ann Intern Med, 1994, 121(12):953 - 959.

[2] Straus S E , Dale J K, Wright R ,et al. Allergy and the chronic fatigue syndrome. J Allergy Clin Immunology,1988:7912795. 